Results Male patients were found to be less likely to develop pain during colonoscopy (odds ratio (OR) 0.43 , 95\% CI $0.35-0.53$, $\mathrm{p}<0.001$ ). Those who had any malignancy in the past (OR 0.69 , $95 \%$ CI $0.49-0.97, p=0.02$ ) and previous abdominal surgery (OR $0.63,95 \%$ CI $0.47-0.84, p=0.02$ ) were less prone to having pain. Better bowel preparation improved the comfort of the procedure (OR 0.67, 95\% CI 0.54-0.85, p = 0.001) however those who used Moviprep as bowel preparation were more likely to complain of pain (OR 1.83, 95\% CI 1.34-2.49, p < 0.001). Higher dose of pethidine requirement was found to be associated with increase likelihood of reporting pain (OR 1.03, 95\% CI 1.02-1.04, p < 0.001) but no association was found with the use of midazolam or buscopan. High performance endoscopists were found to cause less pain (OR $0.35,95 \%$ CI $0.27-0.46, p=p<0.001)$. The presence of diverticular disease showed a strong trend towards increasing probability of pain although it did not reach statistical significance (OR1.4, 95\% CI 1.1-1.8, $p=0.07)$. Age and medical co-morbidities like rheumatological and neurological problems did not have any significant association.

Conclusion Likelihood of having abdominal pain during colonoscopy was found to be associated with being female, having poor bowel preparation and the procedure being performed by non-high performing endoscopists. Patients with past history of malignancy were also noted to have less tendency of having pain. The association of higher dose of pethidine and reported pain was likely to reflect the need of larger doses in such situation. Interestingly, history of previous abdominal surgery did not increase the likelihood of reporting pain and in fact had the opposite effect. The reason for why patients who had Moviprep as bowel preparation were more likely to complain of pain is unknown and this may need to be explored in future studies.

Disclosure of Interest None Declared.

\section{PWE-070 THE DUODENAL-JEJUNAL BYPASS SLEEVE (ENDOBARRIER GASTROINTESTINAL LINER) FOR WEIGHT LOSS AND TREATMENT OF TYPE II DIABETES}

doi:10.1136/gutjnl-2013-304907.359

'S Patel, 'D Hakim, ${ }^{3} \mathrm{~J}$ Mason, ${ }^{4,}$ N N Hakim. ' South Thames Foundation School, London, UK; ${ }^{2}$ Royal College of Surgeons in Ireland, Dublin, Ireland; ${ }^{3}$ Gastroenterology, Trafford General Hospital, Manchester; ${ }^{4}$ mmperial College Healthcare NHS Trust, London, UK

Introduction The Duodenal-jejunal bypass sleeve (EndoBarrier Gastrointestinal Liner) is an endoscopically and fluoroscopically inserted implant designed to aid weight loss, treat type II diabetes mellitus and improve the cardiovascular risk profile of subjects. We aimed to trial this device in a cohort of patients to assess efficacy.

Methods We implanted the EndoBarrier bypass sleeve into 57 patients from January 2011 to December 2012. The EndoBarrier is an impermeable fluoropolymer sleeve that is reversibly fixated to the duodenal bulb and extends $80 \mathrm{~cm}$ into the small bowel, usually terminating in the proximal jejunum. It is implanted in the GI tract endoscopically to create a barrier between food and the wall of the intestine and to delay the mixing of digestive enzymes with food. It alters the activation of hormonal signals that originate in the intestine, thus mimicking the effects of a Roux-en-Y gastric bypass procedure without surgery.

Results Results showed weight loss in all patients, as well as lowering of blood sugar levels. Only 1 early device removal (due migration) occurred. There were no major postoperative side effects.

Conclusion Results confirm that the device reduces blood sugar levels and triggers weight loss. This non-permanent device implanted and removed endoscopically, controlled blood sugar and weight loss without the trauma of surgery. Clinical trials to date, involving more than 300 patients, have demonstrated significant weight loss and diabetes improvement with the Endobarrier.
However, since this is a new procedure and due to the lack of data, it is not yet known if weight loss and diabetes benefits will persist. Disclosure of Interest None Declared.

\section{PWE-071 SAFE SEDATION IN ENDOSCOPY: TIME FOR A NEW APPROACH?}

doi:10.1136/gutjnl-2013-304907.360

1."N Zakeri, 'S Webster, 'S Coda, 'A Humphries, ' $\mathrm{A}$ V Thillainayagam. 'Imperial College Healthcare NHS Trust, London, UK

Introduction Despite increasing awareness of the potential hazards of endoscopic sedation, complications from sedation remain a major concern. Serious harm or death resulting from sedation overdose is now a Department of Health 'never event'1. Previous work by our group (2000-2005) demonstrated a steady sedation reversal incidence of $0.27 \%$. Trust guidelines, in line with the current British Society of Gastroenterology safe sedation guidelines $(2003)^{2}$, were developed in 2005 (revised 2011). We present the results of a retrospective survey, evaluating whether the incidence and risk factors for sedation reversal events have changed.

Methods Our Trust is a large tertiary referral endoscopic centre across 3 sites. A retrospective analysis of all endoscopy $(n=73,989)$ was performed, including all sedated endoscopic procedures carried out between 2007 and $2012(\mathrm{n}=52,553)$. Flumazenil or naloxone administration was used as a marker of sedation overdose requiring reversal. The results were compared to the previous single-site audit of 2000 to 2005 ( $n=20,569)$. Reversal episodes were analysed for associations with total sedation dose given, patient ASA grade, age and procedure undertaken. Statistical analysis was carried out using chi squared test and the linear regression model (Origin ${ }^{\circledR}$ ).

Results In total 149 sedation reversals were recorded, representing $0.28 \%$ of all sedated endoscopic procedures, with no significant difference from the reversal rate $(0.27 \%)$ recorded between 2000 and 2005 ( $p=0.79)$. Mean dose of midazolam used in reversal events was $3.1 \mathrm{mg}$ (range $0.5-14 \mathrm{mg}$ ). Mean dose of opioid (as pethidine equivalent) was $47.9 \mathrm{mg}$ (range $12.5-150 \mathrm{mg}$ ). Higher than recommended doses of midazolam $(5 \mathrm{mg})$ or opioid (pethidine equivalent $50 \mathrm{mg}$ ) were administered in $7.4 \%$ and $6.7 \%$ of reversal events, respectively. Endoscopic Retrograde Cholangiopancreatography (ERCP) was most associated with sedation reversal (1\%). Mean dose of midazolam varied by procedure type and was highest for ERCP (5.1 $\pm 2.9 \mathrm{mg})$ and lowest for flexible sigmoidoscopy $(1.7 \pm 0.6 \mathrm{mg}$; $\mathrm{p}<0.01)$. Mean dose of pethidine or opioid equivalent was highest for ERCP $(78 \pm 38.7 \mathrm{mg}$ ) and lowest for colonoscopy $(33 \pm 13.4 \mathrm{mg}$; $p<0.01)$. Sedation reversal was positively associated with increasing patient ASA grade $(\mathrm{p}<0.05)$.

Conclusion Despite the emergence of national and local guidelines, aimed at safe sedation practise, there was no decline in our Trust's rate of sedation reversals over the last 12 years. Furthermore, the findings suggest there is a subgroup of patients, and a subset of endoscopic procedures, which still carry a significant risk of oversedation requiring reversal. This may support the growing interest in alternative sedation strategies for prolonged therapeutic endoscopic procedures such as ERCP.

Disclosure of Interest None Declared.

\section{REFERENCES}

1. DOH 'Never Events' 2012/13

2. Safety and Sedation During Endoscopic Procedures, BSG 2003.

\section{PWE-072 EFFECT OF CHROMOENDOSCOPY ON ADENOMA DETECTION IN THE COLON: A META-ANALYSIS}

doi:10.1136/gutjnl-2013-304907.361

1,2, $\mathrm{N}$ Mohammed, 1,2V Subramanian. ' Gastroenterology, Leeds Teaching Hospitals NHS Trust; ${ }^{2}$ Leeds Institute of Molecular Medicine, Leeds, UK 
Introduction Early adenoma detection is shown to reduce mortality from colorectal cancers. Advances in endoscopy are aimed at improving adenoma detection. Contrast enhancement using dye spray is reported to improve the detection of subtle mucosal changes. We aim to perform a meta-analysis to look at the effect of chromoendoscopy on adenoma detection in the colon.

Methods Various electronic databases were searched for articles reporting on detection of polyps during colonoscopy comparing standard while light endoscopy and chromoendoscopy. The pooled mean differences in total number of adenomatous polyps detected, number of right and left sided polyps, advanced and flat adenomas, total number of polyps and number of $<5 \mathrm{~mm}$ polyps detected was calculated. A fixed effects model was used unless there was significant heterogeneity. Publication bias was assessed using Funnel plots and Egger's test and heterogeneity was assessed using Cochran's $Q$ and the $\mathrm{I}^{2}$ test.

Results 3714 number of patients from 14 studies were included in the analysis. 7 studies were randomised controlled trials and 7 had a tandem colonoscopy study design. Chromoendoscopy detected significantly higher number of adenomas, advanced adenomas, right and left sided adenomas (Table 1). A significantly higher number for hyperplastic and small $(<5 \mathrm{~mm})$ polyps were detected by chromoendoscopy but no differences were noted for detection of flat adenomas. A random effects model was used because there was significant heterogeneity between the studies. There was some publication bias noted on the funnel plot with fewer number of smaller negative studies included. Sensitivity analysis for publication bias using the trim and fill method which did not change the statistical significance of the pooled analysis.

\section{Abstract PWE-072 Table 1}

\begin{tabular}{lll}
\hline Variable & Pooled mean difference & P value \\
\hline Adenoma detection & $0.139(95 \% \mathrm{Cl} 0.082$ to 0.195$)$ & 0.0001 \\
Right sided adenomas & $0.137(95 \% \mathrm{Cl} 0.048$ to 0.226$)$ & 0.003 \\
Left sided adenomas & $0.117(95 \% \mathrm{Cl} 0.007$ to 0.227$)$ & 0.036 \\
Advanced adenomas & $0.105(95 \% \mathrm{Cl} 0.017$ to 0.194$)$ & 0.019 \\
Flat adenomas & $0.154(95 \% \mathrm{Cl}-0.084$ to 0.393$)$ & 0.205 \\
Hyperplastic & $0.364(95 \% \mathrm{Cl} 0.281$ to 0.447$)$ & 0.001 \\
<5mm & $0.271(95 \% \mathrm{Cl} 0.172$ to 0.369$)$ & 0.001 \\
\hline
\end{tabular}

Conclusion Conclusions: Chromoendoscopy improves detection rate of adenomatous polyps compared to conventional white light endoscopy. This seems greater for advanced as well as right sided adenomas, but significantly higher number of hyperplastic and small $(<5 \mathrm{~mm})$ polyps were detected by chromoendoscopy. Future work needs to focus on the cost effectiveness of chromoendoscopy taking into account the increased time and cost of chromoendoscopy and long term outcomes like reduction in colorectal cancer rates between chromoendoscopic and conventional colonoscopy surveillance.

Disclosure of Interest None Declared.

\section{Inflammatory bowel disease}

\section{PWE-073 THE MULTIDIMENSIONAL NATURE OF IBD FATIGUE: A SYSTEMATIC REVIEW AND META- ANALYSIS}

doi:10.1136/gutjnl-2013-304907.362

1,"I Nathan, 'K C Fragos, 'A Forbes. 'Gastroenterology and Nutrition, University College London, London, UK

Introduction Fatigue is recognised as a troublesome symptom for patients with IBD. There is no consensus regarding mechanisms driving fatigue or treatment. The aim of the present study is to assess the factors associated with IBD fatigue, using a systematic literature review and meta-analysis.
Methods A systematic literature review was performed in PubMed and EMBASE and additional articles and abstracts were identified by a hand search. All studies reporting correlation coefficients (CCs) of fatigue with disease activity were included. A random-effects model was employed to produce a pooled estimate of CCs, which was the effect size in the current analysis. Fisher's Z transformation of the CCs was utilised for the analysis. Publication bias was assessed with funnel plots and Egger's test. All computations were executed with Stata 10.0 and MetaWin.

Results 23 studies were eligible for inclusion (total sample size $=5980)$. Fatigue was strongly correlated with IBD disease activity (pooled CC 0.386 ; $95 \%$ CI 0.335 to 0.435 , range 0.165 to 0.540 ). Although there was no publication bias (failsafe $\mathrm{N}=958$ ), there was moderate heterogeneity $\left(\mathrm{Q}=33.74, \mathrm{df}=14, \mathrm{p}=0.002 ; \mathrm{I}^{2}=58.5 \%\right)$. Subgroup analysis showed a relationship between the correlation of fatigue and disease activity with population group (adult, paediatric) but not with country. Meta regression with percentage of males and mean age did not suggest that either were significant moderators of the particular correlation coefficient. Fatigue was also strongly correlated with quality of life (pooled CC $-0.534 ; 95 \%$ CI -0.636 to -0.414 ), psychological distress (pooled CC $0.515 ; 95 \%$ CI 0.412 to 0.606 ) and daytime sleepiness (pooled CC 0.400; 95\% CI 0.32 to 0.471 ), and was moderately correlated with anaemia (pooled CC $0.167 ; 95 \%$ CI 0.104 to 0.231 ) and social and functional impairment (pooled CC 0.288 ; $95 \%$ CI 0.133 to 0.424 ). Flare ups were not a significant predictor of fatigue $(p>0.05)$. Heterogeneity was present in all analyses $(p<0.05)$, except for the meta-analyses with anaemia, daytime sleepiness and social and functional impairment. Publication bias (assessed with the funnel plot) was more probable in the secondary factors compared with disease activity analyses in the initial correlation.

Conclusion A number of factors were found to correlate with IBD fatigue. These include: psychological distress, daytime sleepiness, disease activity, anaemia and social and functional impairment. This suggests both cognitive and physical aspects of IBD fatigue and clearly highlights the multidimensional nature of this symptom.

This is part of a larger study conducted in collaboration with Crohn's and Colitis UK, King's College London and Addenbrookes' NHS Trust, funded by the Big Lottery Fund.

Disclosure of Interest None Declared.

\section{PWE-074 HOW OFTEN DO WE DISCONTINUE MAINTENANCE INFLIXIMAB DUE TO CLINICAL REMISSION IN CROHN'S DISEASE?}

doi:10.1136/gutjnl-2013-304907.363

1,“J P Seenan, 'J Munro, 'S Laird, 'J W Winter, 'D R Gaya, 'J Morris. 'Gastroenterology, Glasgow Royal Infirmary, Glasgow, UK

Introduction Biologics are increasingly used in the management of Crohn's disease (CD). NICE guidelines advise reassessing CD patients after 12 months of treatment with infliximab and discontinuing therapy for patients in stable clinical remission. Evidence suggests that sustained remission may be achieved in up to $50-60 \%$ of these patients on withdrawal of treatment. However, it is recognised that decisions regarding continuing treatment must be individualised and take into account previous disease behaviour. We sought to determine how often in clinical practise maintenance infliximab was discontinued due to clinical remission.

Methods All patients treated with infliximab for CD between September 2006 and December 2012 were identified from our inflammatory bowel disease (IBD) database. Dates of initiation and termination of treatment were recorded along with the reason for discontinuation. Patients continuing on infliximab were analysed in more detail. Patient demographics, past medical history, Montreal classification, baseline investigations, Harvey-Bradshaw index (HBI) and faecal calprotectin (FC) levels were recorded. 